Tong and Yu et al., Afr J Tradit Complement Altern Med. (2016) 13(1):95-98

http://dx.doi.org/10.4314/ajtcam.v13i1.13

\title{
MICROORGANISMS FROM HANDS OF TRADITIONAL CHINESE MEDICAL DOCTORS IN A CENTRAL HOSPITAL ENVIRONMENT
}

\author{
Ye Sun, LiXiang Yu, Min Sun, YanQing Tong. \\ ${ }^{1}$ Department of Nephrology, Affilliated Hospital to Changchun University of Chinese Medicine, \\ Changchun city, Jilin province, China. ${ }^{2}$ Department of Nephrology, Affilliated Hospital to \\ Changchun University of Chinese Medicine, Changchun city, Jilin province, China. ${ }^{3}$ Department of \\ Nephrology, Affilliated Hospital to Changchun University of Chinese Medicine, Changchun city, \\ Jilin province, China. \\ Corresponding author Email: tongyanqing@yahoo.com
}

\begin{abstract}
Background: In a central hospital, the heavy clinical workload makes one to overlook its hazard to health and can to a large extent promote the transmission of pathogenic microorganisms. It is not uncommon however, to observe practices that deviate from normal standards of hygiene. Hand contact between doctors of TCM and patients may lead to nosocomial infections.

Materials and Methods: Samples were sourced and collected from hands of TCM doctors in a central hospital. The fungal isolates were identified by colonial morphology and microscopic examination. Sensitivity test were performed on each of the identified isolates using the Multiple Disc Diffusion method.

Results: A total of 265 samples were collected from the fingers, palm and back of hands of TCM doctors. Seven organisms were isolated, with $S$. aureus being the most common bacterium with frequency of $55.9 \%$ in the hospital environment. Other bacteria isolated included $P$. aeruginosa, E. coli and $K$. aerogenes. S. cerevisae was the most common fungus isolated with frequency of $61.8 \%$. Fungi isolated included $P$. chrysogenum and A. niger.

Conclusion: The study showed clearly that hand contact between TCM doctors and patients may lead to infection of exposed susceptible patients. Results of antibiotic susceptibility pattern show that isolates in this study are mostly resistant to augmentin and most antibiotics of old generation, while gentamycin and pefloxacin were highly active against most of isolates. Considering the possibility of transmission of contamination, special attention should be directed towards disinfection of TCM doctors' hands.
\end{abstract}

Keyword: Hospital Environment; Traditional Chinese medicine; Microorganism

\section{Introduction}

There is increase in concern about the role of the environment as a reservoir for multi-resistant organisms (Abdollahi et al., 2010). Various researchers have found that these organisms can survive for a long period of time on dry surfaces. While it is difficult to establish whether patients develop an infection as a result of the exposure to these organisms; significant bioburden exist in the environment, and the potential for transmission of disease from reservoirs to patients or health care workers via hands exists (Annand et al., 2009).

Traditional Chinese medicine (TCM) is a system of healing that originated thousands of years ago. It has evolved into a well-developed, coherent system of medicine that uses several modalities to treat and prevent illness (Lao, 1999). The use of touch in treatment (e.g. Tui Na or Chinese massage) and making a diagnosis (e.g. pulse feeling) in TCM is integral. The art of touch in TCM is highly sophisticated and includes the palpation of areas of pain and diagnostic points and the feeling of the patient's pulse. However, the hand contact between doctors of TCM and patients is seen against the background rise in the rate of nosocomial infections.

In a central hospital, the heavy clinical workload makes one to overlook its hazard to health and can to a large extent promote the transmission of pathogenic microorganisms. It is not uncommon however, to observe practices that deviate from normal standards of hygiene. The constant contacting between TCM doctors and patients makes it open for arrays of microorganisms, making it a harbour and a breeding ground for microbes especially those associated with the skin, from where different microorganisms are spread from one to another.

The objective of this study is aimed at (i) detecting the level of microbial contamination of TCM doctors' hands using appropriate cultural and biochemical techniques for microbial identification; (ii) to determine the antibiotic susceptibility of the microorganisms isolated; (iii) establishing a link between TCM doctors' hands and nosocomial infection.

\section{Materials and Methods Collection of Samples}

Samples were sourced and collected from hands of TCM doctors in a central hospital, the Affiliated Hospital to Changchun University of Chinese Medicine. Two hundred and sixty-five (265) samples were collected. Sample from each hand was collected using sterile cotton swab stick. The swab sticks were moistened with sterile normal saline and used to swab the fingers, palm and back of hands. 


\section{Tong and Yu et al., Afr J Tradit Complement Altern Med. (2016) 13(1):95-98 http://dx.doi.org/10.4314/ajtcam.v13i1.13 \\ Incubation and Identification}

Serial dilution was undertaken using sterile distilled water and the inoculated normal saline. $9 \mathrm{ml}$ distilled water was dispensed into four test tubes for each sample. Using a sterile syringe, $1 \mathrm{ml}$ of the inoculated normal saline was taken and added to the first $9 \mathrm{ml}$ of sterile distilled water $\left(10^{-1}\right)$ and mixed. $1 \mathrm{ml}$ was withdrawn and added to the next $9 \mathrm{ml}$ of sterile distilled water and mixed $\left(10^{-2}\right)$. This was repeated to get $10^{-3}$ and $10^{-4}$. One milliliter from the fourth tube of the already diluted sample was poured into a sterile petri dish and nutrient agar was added and mixed using clockwise and anti-clockwise movements. It was allowed to set and incubated for 24 hours at $37^{\circ} \mathrm{C}$ for bacteria, and 48 hours for fungi at room temperature. Colonies were counted after 24 hours and 48 hours for bacteria and fungi respectively. Samples from the swab sticks were cultured onto MacConkey agar, Nutrient agar, Blood agar and Potato Dextrose agar.

All bacteria were identified by standard procedures (Edwards et al., 1955). This was done on the basis of cultural appearance of colonies, morphology and by conventional biochemical tests. The lactose and non-lactose fermenting organisms on MacConkey agar plates were subjected to biochemical tests and other tests for identification purpose including gram reaction, motility, indole, citrate, urease, and oxidase.

The fungal isolates were identified by colonial morphology and microscopic examination. It was done through macroscopic observation of the colonies and microscopically by examination of the micro-structural characteristics and a comparative analysis with parameters established in the conventional taxonomy, in accordance with the revised literature (Hoog et al., 1995).

\section{Antibiotic Sensitivity}

Sensitivity test were performed on each of the identified isolates using the Multiple Disc Diffusion method. The organisms already inoculated into a Bijou bottle containing sterile peptone water were used to flood the surface of solid Muller- Hinton agar and after 3 seconds, allowed to drain. A multiple disc containing the antibiotics was placed on the surface of the agar. The multi disc used depends on the gram reaction of the organisms. The agar plate was allowed to stay on the bench for 15 minutes to allow proper absorption of the antibiotics before it was incubated at $37^{\circ} \mathrm{C}$ for 24 hours. After the 24 hours of incubation, bacterial lawns were observed on the plate. Zones of inhibition of bacterial growth were present around the antibiotic disc. The sensitivity of the test organisms to a particular antibiotic was scored by measuring the zone of inhibition around each of the antibiotic in the multiple discs.

\section{Results \\ Isolation of bacteria and fungi}

Out of a total of 265 samples collected from TCM doctors' thenar area of hands in hospital environment, bacterial growth was recorded in $153(57.7 \%)$. The average total aerobic bacterial counts recorded were $4.25 \times 10^{5} \mathrm{cfu} / \mathrm{ml}$. Fungi growth was found in 34 (12.8\%) with an average fungi count of $3.10 \times 10^{5} \mathrm{cfu} / \mathrm{ml}$.

Prevalence and occurrences of the bacterial genera isolated were also studied. The result showed that Staphylococcus aureus, Pseudomonas aeruginosa, Escherichia coli and Klebsiella aerogenes were the isolates recorded. The frequency of occurrence showed that $S$. aureus was most frequently isolated $(\mathrm{n}=85,55.9 \%)$ in the hospital environment, followed by P.aeruginosa and K. aerogenes which had prevalent rates of $22.4 \%(\mathrm{n}=34)$ and $15.1 \%(\mathrm{n}=23)$ respectively. E. coli were the least frequent isolate recovered $(\mathrm{n}=11,7.2 \%)$.

The distribution of fungi isolated indicated that Saccharomyces cerevisae had the highest frequency of occurrence from the environment. The percentage of S. cerevisae isolated was $61.8 \%(\mathrm{n}=21)$. Other fungi isolated were Penicillium chrysogenum and Aspergillus niger $(n=13,38.2 \%)$, with $P$. chrysogenum recording the least frequency of occurrence $(n=6,17.6 \%)$.

\section{Antibiotic Sensitivity}

Result of the antibiotic susceptibility pattern as shown in Table 1 indicates that isolates from the study were most resistant to Augmentin and Ampiclox. The isolates were most susceptible to Gentamycin. 71.2\% of isolates from hands of TCM doctors showed resistance to Augmentin and Ampiclox, which were followed by Erythromycin (58.5\%), Septrin (57.5\%), Sparfloxacin (55.6\%), while Gentamycin recorded the least resistance $(5.9 \%)$.

\section{Discussion}

This study was to identify the isolation of microbial flora found on hands of TCM doctors in the hospital environment, in the belief that the numbers and types of microorganism as well as the antibiotic susceptibility pattern would have as a valid basis for conclusions as to the sanitary risk there may be by hand contact with patients. The collection of samples was made in the central hospital environment (Affiliated Hospital to Changchun University of Chinese Medicine).

Bacteria isolated in increasing order include, E. coli, K. aerogenes, P. aeruginosa and S. aureus. The occurrence of $S$. aureus was the highest of all the bacterial isolates. This may be due to its resistance to drying which favours its transmission, and its presence as part of the normal flora of the nose, mouth and skin (Gould et al., 2010). Its transmission from one host to another susceptible host is known to be responsible for epidemic pyogenic infections in the hospitals as well as epidemic diseases. However, this study agrees with earlier work conducted by other researchers who reported that $S$. aureus make up to $20 \%$ of the microbial load in the air. They also reported that the organism is constantly disseminated from the nasal cavity during breathing, talking and even exercise (Datta et al., 2009). Staphylococcus spp. is also known to cause illnesses ranging from pimples and boils to 
Tong and Yu et al., Afr J Tradit Complement Altern Med. (2016) 13(1):95-98

http://dx.doi.org/10.4314/ajtcam.v13i1.13

Table 1: Antibiotic Susceptibility Pattern of Bacterial Isolates

\begin{tabular}{|c|c|c|c|c|c|}
\hline & Antibiotics & $\begin{array}{l}\text { S. aureus } \\
n=85\end{array}$ & $\begin{array}{l}\text { P. aeruginosa } \\
n=34\end{array}$ & $\begin{array}{l}\begin{array}{l}\text { E. coli } \\
n=11\end{array} \\
\end{array}$ & $\begin{array}{l}\text { K. aerogenes } \\
n=23\end{array}$ \\
\hline \multirow{14}{*}{$\begin{array}{l}\text { Resistance } \\
\text { of Isolates to } \\
\text { Antibiotics } \\
(\text { No } / \%)\end{array}$} & Amoxicillin (30ug) & $43(50.6)$ & $15(44.1)$ & $8(72.7)$ & $11(47.8)$ \\
\hline & Rocephin (30ug) & $52(61.2)$ & $13(38.2)$ & $5(45.5)$ & $6(26.1)$ \\
\hline & Ciprofloxacin (10ug) & $25(29.4)$ & $11(32.3)$ & $3(27.3)$ & $13(56.5)$ \\
\hline & Streptomycin (30ug) & $26(30.4)$ & $18(52.9)$ & $3(27.3)$ & $2(8.7)$ \\
\hline & Septrin (30ug) & $61(71.8)$ & $18(52.9)$ & $6(54.5)$ & $3(13.0)$ \\
\hline & Erythromycin (10ug) & $58(68.2)$ & $19(55.9)$ & $8(72.7)$ & $5(21.7)$ \\
\hline & Pefloxacin (10ug) & $9(10.4)$ & $6(17.6)$ & $0(0)$ & $3(13.0)$ \\
\hline & Gentamycin (10ug) & $5(5.9)$ & $0(0)$ & $\boldsymbol{O}(\boldsymbol{0})$ & $4(17.4)$ \\
\hline & Ampiclox (30ug) & $68(80.0)$ & $17(50.0)$ & $8(72.7)$ & $16(69.6)$ \\
\hline & Zinnacef (20ug) & $43(50.6)$ & $11(32.4)$ & $7(63.6)$ & $10(43.5)$ \\
\hline & Ofloxacin (20ug) & $36(42.4)$ & $9(26.5)$ & $5(45.5)$ & $10(43.5)$ \\
\hline & Chloramphenicol (30ug) & $52(61.2)$ & $19(55.9)$ & $2(18.2)$ & $10(43.5)$ \\
\hline & Augumentin (30ug) & $71(83.5)$ & $18(52.9)$ & $8(72.7)$ & $12(52.2)$ \\
\hline & Sparfloxacin (10ug) & $52(61.2)$ & $18(52.9)$ & $6(54.5)$ & $9(39.1)$ \\
\hline
\end{tabular}

pneumonia and meningitis, a scenario supported by the high population of colony isolates (Brooks et al., 2001).

$P$. aeruginosa has been reported to be the most isolated nosocomial pathogen accounting for $10.1 \%$ of all hospital acquired infections, primarily in immune-compromised individuals (Akinyemi et al., 2009). It is a free living bacterium commonly found in soil and water. However, it occurs regularly on the surfaces of plants and occasionally on the surfaces of animals. P. aeruginosa has become recognized as emerging opportunistic pathogen of clinical relevance. Several epidemiological studies track its occurrence as a nosocomial pathogen and indicate that antibiotic resistance is increasing in clinical isolates (Todar, 2004).

Klebsiella are ubiquitous and may colonize the skin, pharynx or gastro intestinal tract in human. They are commonly passed by hands of hospital personnel. Common sites of nosocomial Klebsiella infections include the urinary tract, lower respiratory tract, biliary tract and surgical wound sites. Fungal colonies occurred in 34 (12.8\%) out of the 265 samples collected from the hospital environment. Fungi isolated include S. cerevisae, P. chrysogenum and A. niger. P. chrysogenum is mentioned as a keratitis and endophtalmitis agent (Coutinho et al., 2007). A. niger has been reported as cutaneous infection agent (Coutinho et al., 2007). S. cerevisae is a well-known microbe used intensively in industrial capacities. Although widely distributed in nature and displaying low virulence against humans, invasive S. cerevisae infections have been increasingly common in recent decades, having been identified in about $4 \%$ of positive fungal blood cultures, including those from immunocompetent subjects (Enache-Angoulvant et al., 2005). It was first described as a pathogen in 1958 by Reihersol and Hoel in a bronchopneumonia patient's serial sputum sample (Enache-Angoulvant et al., 2005). It has been recognized as a transient colonizer of the gastrointestinal mucosa (particularly after the contamination of food), the female genital tract and the respiratory tract. Its access to the body is believed to be predominately gastrointestinal, in spite of reports on catheter contamination due to contaminated hands. The presence of the microbe may therefore be considered a healthcare-related infection (Silva et al., 2011). P. chrysogenum is rarely pathogenic except in extenuating circumstances such as people with severely suppressed immune systems, like those with human immunodeficiency virus (HIV). Symptoms of infection include pulmonary infection including pneumonia, localized granulomas, fungus balls and systemic infection. The most common avenue for the implementation of infection by this organism in the eye is by penetrating trauma. $P$. chrysogenum can also act as an allergen and an asthma inducer (Adrian et al., 2005). Due to lack of inadequate cleaning of TCM doctors' hands, the existing fungi may cause mycosis in people from different social classes and variable habits, especially immune-compromised patients. The latter have greater possibility of becoming infected by the fungal spores and other microorganisms presented. The implication of this observation is that the possibility of being infected with bacteria and fungi pathogens simply by pulse feeling is high. The susceptibility patterns shown in this study suggest that it is absolutely necessary to obtain sensitivity reports before the initiation of antimicrobial therapy in suspected nosocomial patients. It should also be noted that antibiotic sensitivity testing is only a guide and that the conditions in vivo may be different from those obtained in vitro. The ultimate decision to utilize a particular antibiotic depends on such factors as toxicity, costs and serum attainable levels. This study agrees with other studies that Staphylococcus species, besides having established itself as a major hospital pathogen, is now beginning to prevail in a wider community (Smith et al., 2009). Present results show that gentamycin is highly active against most gram negative and positive bacteria. These results are not in concordance with other reports which showed that vancomycin and imipenem are the highly active ones (Japoni et al., 2011). Gentamycin being the least resistant to the isolated bacteria may be due to the fact that it does not have an oral version; it is only in form of injections which reduces its abuse. However, it is not expected that this activity continues for a long time as it has been observed in some studies 


\section{http://dx.doi.org/10.4314/ajtcam.v13i1.13}

Tong and Yu et al., Afr J Tradit Complement Altern Med. (2016) 13(1):95-98

(Japoni et al., 2011) because it is cheap and readily available. The organisms were also sensitive to pefloxacin, which is not a relatively common antibiotic and thereby making it less available for abuse. The isolates show highest resistance to Augmentin which may be as a result of the drug being readily available both in tablet and injection forms and overuse/abuse. However, it is observed that the organisms from the hospital environment are also resistant to most of the old generation drugs (Ampiclox, Erythromycin, Chloramphenicol, Septrin, etc.), which are more available and misused. It has been proposed that frequencies and antibacterial susceptibility patterns of nosocomial pathogens continuously change, which demands periodical surveillance of their fluctuations (Japoni et al., 2011).

\section{Conclusion}

It should be recognized that TCM doctors' hands may be a source for transmission of hospital-acquired infections. Simple measures such as increasing hand hygiene and regular decontamination of hands with alcohol disinfectant/antibacterial wipes may reduce the risk of crosscontamination. Also, strict adherence to infection control and precautions such as hand washing and good hygienic practice among TCM doctors is advocated, to prevent the possibility of hands as vehicles of transmission of both hospital and community-acquired bacterial diseases.

\section{Acknowledgment}

All authors in the article have contributed significantly, and that all authors are in agreement with the content of the manuscript. There is no conflict of interest. The research was funded by the Natural Science Foundation of China (No. 81374039).

\section{References}

1. Abdollahi A, Mahfouzi S. (2010). Bacteria contamination of hospital telephones. Pakistan J Med Sci 26: 747-750.

2. Adrian BL, Burdette SD, Herchline TE. (2005). "Intestinal invasion and disseminated disease associated with Penicillium chrysogenum". Ann Clin Microb Anti 4: 21-24.

3. Akinyemi KO, Atapu AD, Adetona OO, Coke AO. (2009). Mobile phones spread bacterial infections in Nigeria. J Infect Dev Countr 3: 628-632.

4. Annand JW, Bajaj N, Sheth A, Burgess J, Brooke JS. (2009). Potential pathogens and effective disinfectants on public telephones at a large urban United States University. J Environ Health 71: 24-28.

5. Brooks G, Butel J, Morse S. (2001). The staphylococci. In: Medical Microbiology. 22 Ed. McGraw-Hill, 197-203.

6. Coutinho FP, Cavalcanti MS, Neto FC. (2007). Isolation of filamentous fungi from public telephones of the metropolitan region of the city of recife, pe, brazil. Braz J Microbiol 38: 324-329.

7. Datta P, Rani H, Chander J, Gupta V. (2009). Bacterial Contamination of Mobile Phones of Healthcare Workers. Indian J Med Microbiol 27: 279-281.

8. Edwards PR, WH Ewing. (1955). Identification of Enterobacteriaceae. Burgess Publishing Co., Minneapolis, 20-28.

9. Enache-Angoulvant A, Hennequin C. (2005). Invasive Saccharomyces infection: a comprehensive review. Clin Infect Dis 41: 1559-1568.

10. Gould SW, Cuschieri P, Rollason J, Hilton AC, Easmon S, Fielder MD.(2010). The need for continued monitoring of antibiotic resistance patterns in clinical isolates f Staphylococcus aureus from London and Malta. Ann Clin Microbiol Antimicrob 9: 20-25.

11. Hoog GS de, Guarro J. (1995). Atlas of Clinical Fungi. The Netherlands, Centraalbureau voor Schimmelcultures Baarn and Delft, 1-15.

12. Japoni A, Kalani M, Alborzi A, Japoni S, Rafaatpour N. (2011). Comparative study of antibacterial susceptibility patterns of the bacteria isolated from patients' blood samples over two periods. Am J Infect Dis 7: 1-7.

13. Lao L. (1999). Traditional Chinese Medicine. In. Essentials of Complementary and Alternative Medicine. Jonas WB, Levin JS, Eds. Baltimore, Md., Lippincott Williams and Wilkins, 216-232.

14. Silva FHA, Paco FR, Reis E, Amaral V. (2011). Saccharomyces cerevisae infection: an unusual pathogen in ICU. Revista Brasieleira de Terapia Intensiva 23: 108-111.

15. Smith SI, Opere B, Goodluck HT, Akindolire OT, Folaranmi A, Odekeye OM, Omonigbehin EA. (2009). Antibiotic susceptibility pattern of Staphylococcus species. Singap Med J 50: 208-213.

16. Todar, K. (2004) Web Review of Todar's Online Textbook of Bacteriology “The good, the Bad and the Deadly”. Sci Mag 304: 1421-1425. 